\title{
Usage of Fuzzy Logic Method for Tunnel Boring Machines
}

\author{
Omur Acaroglu Ergun \\ Istanbul Technical University, Mining Engineering Department \\ Maslak, Istanbul, Turkey \\ acaroglu@itu.edu.tr
}

\begin{abstract}
Performance prediction of mechanical excavation machines is vitally important to determine whether the machine is proper for the formation being excavated and to define the cost of the project before starting it. There are a number of analytical, empirical and numerical models for this aim. Many engineering and geological systems have many imprecision and uncertainties and exact solution rarely exist. Beside effects of many parameters, dealing with rock makes the estimation of cutting forces and tunnel boring machine's performance prediction problem complex. Alternative methods such as fuzzy logic have become useful to research such problems having uncertainties in recent years. Fuzzy logic approaches provides to evaluate all data without accepting precondition and depending on special conditions. Therefore, this method has found widespread application to solve problems in these systems including mechanical excavation applications In this study, models which are established by fuzzy logic method are explained to estimate the performance prediction parameters of tunnel boring machines such as specific energy, torque and thrust requirement.
\end{abstract}

Keywords: Fuzzy Logic Method, Tunnel Boring Machines, Performance Prediction

\section{Introduction}

Tunnel boring machines (TBMs), which are used for excavation tunnels in nearly all type rock masses and geological conditions in tunneling construction. They have numerous advantages including secure and high advance rate excavation. Furthermore, they do not have the disadvantages of roadheaders which are not applied on unstable or hard rock conditions. TBMs are designed and sized in different configurations depending on the project and geological conditions. They can be classified as hard rock tunneling and soft ground tunneling machines as their size changes from $1.5 \mathrm{~m}$ to $15 \mathrm{~m}$ diameter [1].

Disc cutters are widely used on TBMs and they are capable of cutting a variety rock types with different properties. Early disc cutters had a V-shape (V-profile) which caused a rapid loss of efficiency as the tip wear occurred. Beginning in the late 1970s, V-shape ring profiles were replaced by constant cross section (CCS) profiles to maintain cutting efficiency as tip wore out. Disc cutters having between $49 \mathrm{~cm}$ (19 in.) and $8 \mathrm{~cm}(3$ in.) diameter have been used, generally after $30 \mathrm{~cm}$ (12 in.), they are called as mini disc cutters [2].

Many engineering, mining and geological subjects have imprecision and uncertainties and exact solution may not exist. Studying with rock makes complex of the estimation of cutting forces and TBMs performance prediction problem. Alternative methods such as fuzzy logic have become useful to solve such problems having uncertainties in recent years. This method provides to evaluate all data without accepting precondition and depending on special conditions [3-10].

\section{Cutting Forces Acting On Disc Cutters}

Cutting forces which are called normal force (FN), rolling force (FR) and side force (FS) act on a disc cutter shown in Fig. 1. Normal force is defined as force acting under a disc perpendicularly as it penetrates. Normal force constitutes thrust requirement of a machine to penetrate the disc cutters into the rock at a desired penetration. Thrust requirement of a TBM is estimated by Eq. (1). Rolling force acts on the disc along the cutting direction as seen from Fig. 1. This force determines torque and power requirements of a machine to rotate the cutterhead at a certain penetration of the cutters. Using rolling force, torque requirement and power of a TBM cutterhead are calculated by Eqs. (2) and (3). It is also used to estimate the specific energy requirement of cutters as seen in Eq. (4). By using the SE value obtained from Equation 4, instantaneous penetration rate (IPR) of TBMs can be estimated with Eq. (5). 


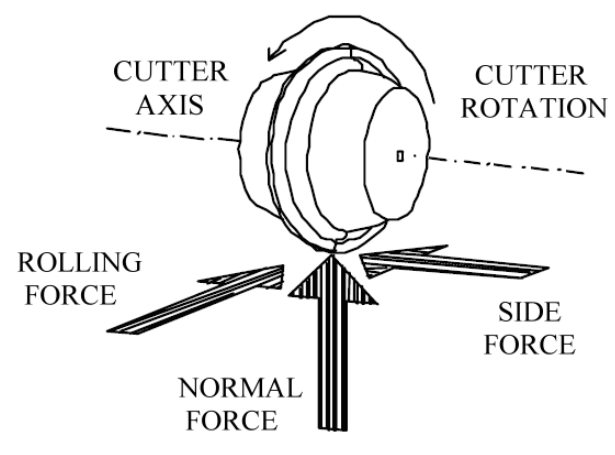

Fig. 1: Cutting forces acting on a disc.

$$
T H=F_{N} \cdot N
$$

Th: Thrust requirement of a TBM Cutterhead

$\mathrm{F}_{\mathrm{N}}$ : Normal force $(\mathrm{kN})$

$\mathrm{N}$ : Number of disc cutter used on the cutterhead

$$
T=\sum_{i=1}^{N} F_{R} \cdot L_{i}
$$

$\mathrm{T}$ : Torque requirement of a TBM cutterhead $(\mathrm{kNm})$

$\mathrm{F}_{\mathrm{R}}$ : Rolling force $(\mathrm{kN})$

L: Distance between center of the cutterhead and i. disc cutter

$\mathrm{N}$ : Number of disc cutter used on the cutterhead

$$
P=2 \pi \cdot M \cdot T
$$

P: Power of a TBM cutterhead $(\mathrm{kW})$

$\mathrm{T}$ : Torque requiremetn of the cutterhead $(\mathrm{kNm})$

M: RPM of the cutterhead

$$
S E=\frac{F_{R}}{S \cdot p}
$$

SE: Specific energy $\left(\mathrm{kW}-\mathrm{hr} / \mathrm{m}^{3}\right)$

$\mathrm{F}_{\mathrm{R}}$ : Rolling force $(\mathrm{kN})$

p: Cutting penetration

S: Spacing between cuts

$$
I R P=\frac{P \cdot k}{S E \cdot A}
$$

IPR: Instantaneous Penetration Rate $(\mathrm{m} / \mathrm{hr})$

P: Power of cutterhead of the machine $(\mathrm{kW})$

SE: Specific energy $\left(\mathrm{kW}-\mathrm{hr} / \mathrm{m}^{3}\right)$ 
$\mathrm{k}$ : Mechanical efficiency factor

A: Tunnel area $\left(\mathrm{m}^{2}\right)$

A number of different models to estimate cutting forces and SE value. Considering spacing (S) and penetration (p) parameters of the cut, rolling force values can be converted to SE values and vice versa. Cutting forces can be also directly measured form the full scale linear cutting test machine (LCM). Models and equations about this subject are based on various parameters that can be classified into three main groups such as rock mechanical properties, disc cutter dimensions and cutting geometry $[1,11-17]$.

\section{Fuzzy Logic Method}

Fuzzy logic which was formalized by Zadeh is a matter of the fuzzy set theory particularly used to dealing with process imprecise information with a changed membership function [18]. In a classical set; an element belongs to, or does not belong to, a set. An element of a fuzzy set naturally belongs to the set with membership values from the interval $[0,1]$. Because fuzzy sets describe vague concepts based on the premise that the elements used are not numbers but belong to words or the value of a linguistic variable. Membership function of fuzzy sets can be either linear (trapezoidal or triangular) or various forms of non-linear, depending on the nature of the system being studied. A triangular fuzzy set of the membership function of is calculated by Equation. 3 [18].

$$
M D_{A}(x)=\left\{\begin{array}{c}
0, x<a \\
\frac{x-a}{b-a}, a \leq x<b \\
\frac{c-x}{c-b}, b \leq x<c \\
0, x>c
\end{array}\right.
$$

$\mathrm{MD}_{\mathrm{A}}(\mathrm{x})$ : Membership function of fuzzy set

$\mathrm{a}, \mathrm{b}, \mathrm{c}$ : Constant

Using by fuzzy conditional rules, fuzzy proposition allows describing input-output relationship. Zadeh introduced the inference mechanism of fuzzy logic reasoning, which based on the compositional rule of inference. By using this inference mechanism, an output set is obtained given the rules and the input variables. A fuzzy conditional rule is generally made up a premise and a consequent part (IF premise THEN consequent), for example, 'if the level1 high, then level2 low', where the terms high and low can be represented by fuzzy sets or, more specifically by membership functions [19].

Mamdani and Assilian [20] showed that the concepts of fuzzy sets and fuzzy logic can translate an entirely unstructured set of linguistic heuristics into an algorithm. This algorithm is one of the most used fuzzy methods to apply in complex engineering geological problems, since most geological processes are defined with linguistic variables or simple vague predicates. The Mamdani fuzzy algorithm takes the following form;

$$
\text { If } X_{l} \text { is } A_{i l} \ldots \text { and } X_{r} \text { is } A_{\text {ir }} \text { then } Y \text { is } B_{i} \text { for } l=1,2, \ldots K
$$

$\mathrm{X}_{\mathrm{l}}, \mathrm{X}_{\mathrm{r}}$ : Input variables

$\mathrm{A}_{\mathrm{il}}, \mathrm{A}_{\mathrm{ir}}, \mathrm{B}_{\mathrm{i}}$ : Linguistic terms (fuzzy sets)

Y: Output variables, K: Number of rules

In the Mamdani fuzzy model, different fuzzy set operators, such as "and", "or" and "not", can be used to combine the premise propositions of the rules. In this algorithm, the contribution of each rule to the output of the model is a fuzzy set. The inference reasoning mechanism in the Mamdani fuzzy model is based on the compositional rule of inference.

\section{Fuzzy Logic Models For TBM's Performance Prediction}

In this study, developed fuzzy logic model, which predicts normal force (FN) and SE value of a CCS disc cutter, is defined. In order to estimate FN and SE value, rock mechanical properties such as Uniaxial Compressive Strength (UCS) and Brazilian Tensile Strength (BTS), disc dimensions such as disc diameter (DD) and tip width (DTW) and cutting 
geometry such as spacing (S) and penetration (p) are used as input parameters. FN and SE parameters are used as output variables. It is based on experience and the data which has been set by Earth Mechanic Institute of Colorado School of Mines performing LCM test for about 30 years $[9,10]$.

Input and output variables are shown in Figure 2 for FN Model and in Figure 3 for SE Model. Fuzzy Inference System (FIS) Editor was used to establish input and output variables. Each input variable and output variable were fuzzified with membership functions which define how each point in the input and output space is mapped to a membership value (or degree of membership) between 0 and 1. Both input and output variables were fuzzy proposition in Mamdani Model. Range of the input and output variables and their membership functions used in the models are given in Table $1[9,10]$.

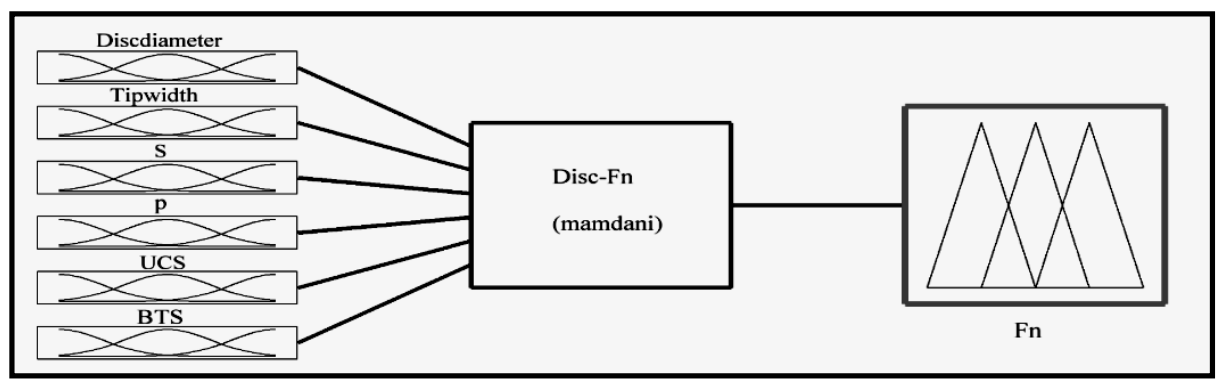

Fig. 2: Input variables and output of the FN model.

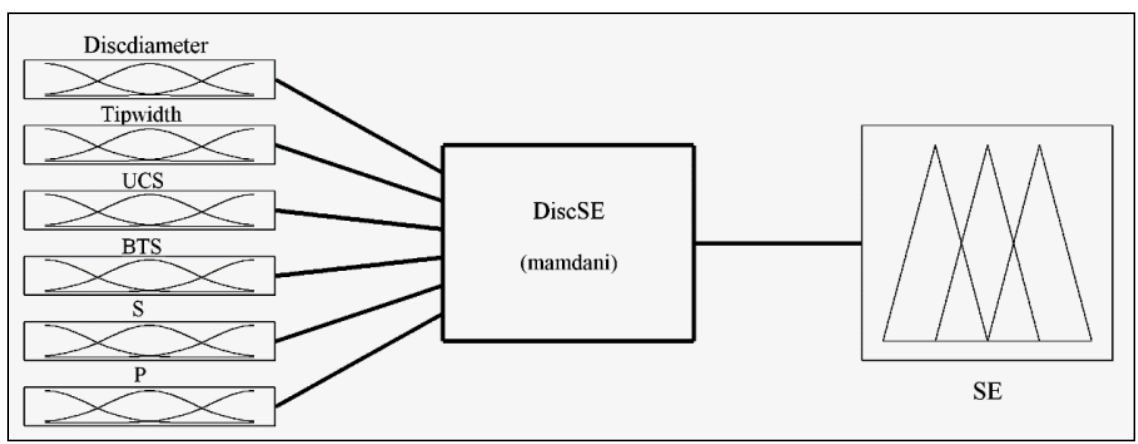

Fig. 3: Input variables and output of the SE model.

Table 1: Range of the input and output variables and their membership functions used in the models.

\begin{tabular}{|l|l|l|}
\hline Input/Output Variables & Ranges & Membership Functions \\
\hline Disc Cutter Diameter & $5-51 \mathrm{~cm}$ & small, medium and big \\
\hline Disc Cutter Width & $0.5-2.3 \mathrm{~cm}$ & thin, medium and thick \\
\hline Spacing between discs & $0.5-18 \mathrm{~cm}$ & small and high \\
\hline Penetration of disc & $0.01-4.05 \mathrm{~cm}$ & very low, low, medium, high and very high \\
\hline Rock Compressive Strength & $21-310 \mathrm{MPa}$ & poor, fair and good \\
\hline Rock Tensile Strength & $1.7-21 \mathrm{MPa}$ & poor and good \\
\hline Normal Force & $1.35-445 \mathrm{kN}$ & $\begin{array}{l}\text { very very very low, very very low, very low, low, medium, high, } \\
\text { very high, very very high and very very very high }\end{array}$ \\
\hline Specific Energy & $0.01-34 \mathrm{~kW}-\mathrm{hr} / \mathrm{m} 3$ & $\begin{array}{l}\text { very very very low, very very low, very low, low, medium, high, } \\
\text { very high, very very high and very very very high. }\end{array}$ \\
\hline
\end{tabular}

The fuzzy proposition need to be represented by an implication function to conduct inference in a rule based fuzzy model. The implication function is called a fuzzy if-then rule or a fuzzy conditional statement. The fuzzy if-then rule takes the following general form: 


\section{If $\mathrm{X}$ is $\mathrm{A}$ then $\mathrm{Y}$ is $\mathrm{B}$}

Where, A and B are linguistic values represented by fuzzy sets. The use of fuzzy set provides the generalization of the information used to describe the behaviour system. The "if" part of the rule is called premise and the "then" part of the rule is called consequence. A total of 324 rules and 540 rules were utilized SE and FN Model respectively. Decision from combinations of input membership functions (premise part) to output membership functions (consequent part) was made by experience and considering data set $[9,10]$.

Values from the SE model and Equation 5, instantaneous penetration rate of TBM's could be estimated. After the SE value which is obtained from the model and $S$ and $p$ are put into Equation 4, the FR value can be obtained easily. It is known that SE value can be converted to Rolling force by using Equation 4 and vice versa. As putting FR value into Equation 2, torque requirement can be obtained. FN values obtained from the model can be used to define the thrust requirement of TBMs using Equation 1 [9, 10].

The FN, SE and FR results obtained from the models and real values of them obtained from LCM were compared to verify the models. The graphics in Fig. 4, Fig. 5 and Fig. 6 shows result of the model and real value comparisons. Values of models are very close the line of $y=x$ means that they are not away from real values with high correlation values as seen from the graphics.

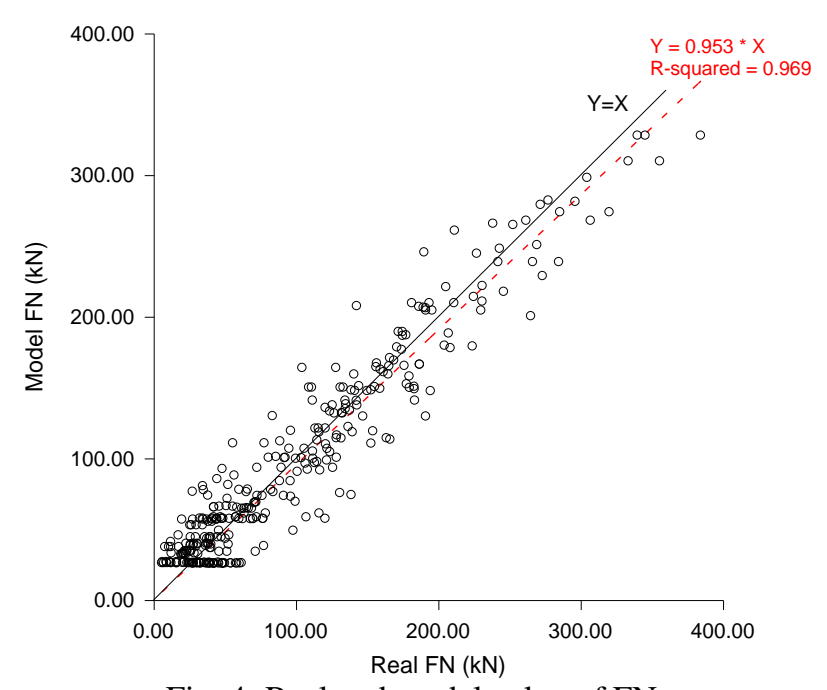

Fig. 4: Real and model value of FN.

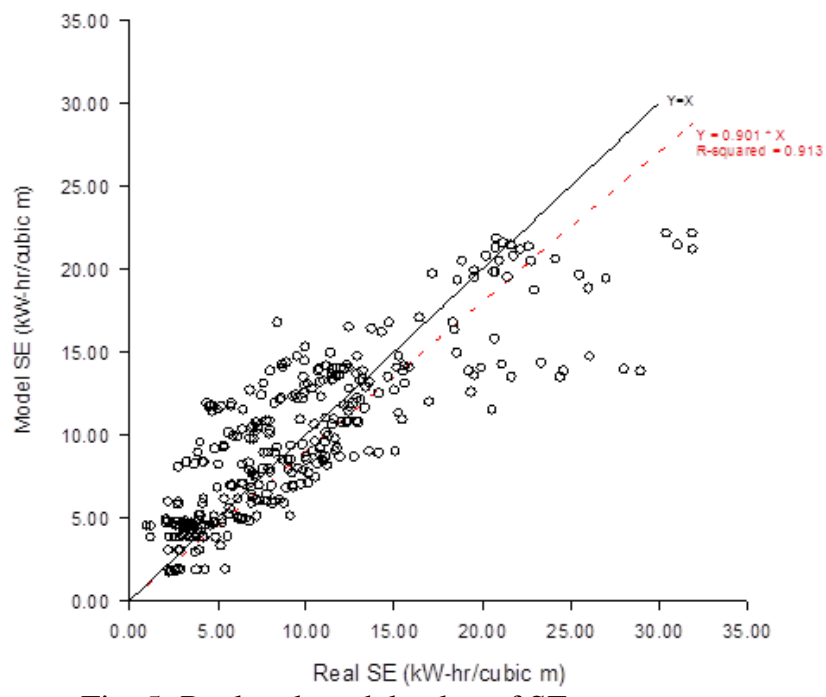

Fig. 5: Real and model value of SE. 


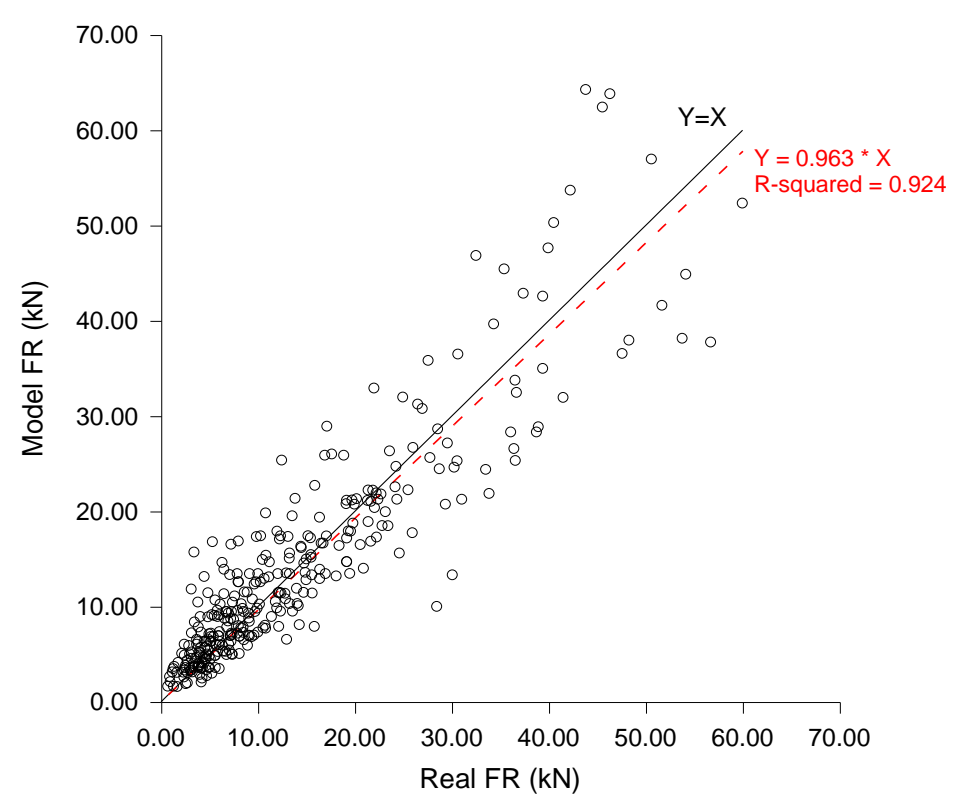

Fig. 6: Real and model value of FR.

\section{Conclusions}

Introduction and application of disc cutters on tunnel boring machines have allowed the excavation of harder materials, resulting in a wider use of these machines in underground constructions. However, successful application of tunnel boring machines technology depends on accurate performance prediction of these machines for determining project schedule and costs.

By using fuzzy logic method, two model was established to predict specific energy, thrust and torque requirement of tunnel boring machines. The comparison of the real value and model value shows that the model values are close the real values. Like these model predictions could be made without any boundary limitations.

Fuzzy logic method has increased to apply on almost all research area particularly including complexity and uncertainty and variables can be modelled considering many possible variables affecting the system. Fuzzy logic approaches provides to evaluate all data without accepting precondition and depending on special conditions.

\section{References}

[1] I. R. Murhead and L. G. Glossop, "Hard rock tunnelling machines," IMM, London, 1968.

[2] J. E. Friant and L. Ozdemir, "Development of the high thrust mini-dic cutter for microtunnelling applications," NoDig Eng., pp. 12-16, 1994.

[3] M. A. Grima, P. N. W. Verhoef, "Forecasting rock trencher performance using fuzzy logic," International Journal of Rock Mech.\&Mining Sci.\&Geomech., vol. 36, no. 4, pp. 413-432, 1999.

[4] M. A. Grima, P. A. Bruines, P. N. W. Verhoef, "Modelling tunnel boring machine performance by neuro-fuzzy methods," Tunn. and Undergr. Space Technol., vol. 15, no. 3, pp. 259-269, 2000.

[5] O. Acaroglu, H. Ergin and S. Eskikaya, "The use of fuzzy logic methods to predict specific energy for roadheaders," in 10th International Symposium on Mine Planning and Equipment Selection, New Delhi, India, pp. 173-179, 2001.

[6] O. Acaroglu, "Stability analysis of the boom type tunnelling machines and optimum selection," Ph.D. dissertation, Istanbul Technical University, Turkey, 2004.

[7] T. Kim, "Development of a fuzzy logic based utilization predictor model for hard rock tunnel boring machines," Ph.D. dissertation, T-5946 Colorado School of Mines, USA, 2004.

[8] O. Acaroglu, C. Ferigunoglu and D. Tumac, "Selection of roadheaders by fuzzy multiple attribute decision making method," IMM Mining Technology, vol. 115, no. 3, pp. 1-8, 2006.

[9] O. Acaroglu, L. Ozdemir and B. Asbury, "A fuzzy logic model to predict specific energy requirement for TBM performance prediction," Tunnelling and Underground Space Technology, vol. 23, no. 5, pp. 600-608, 2008. 
[10] O. Acaroglu, "Prediction of Thrust And Torque Requirements of TBMs With Fuzzy Logic Models," Tunneling and Underground Space Technology Incorporating Trenchless Technology Research, vol. 26, no 2, pp. 267-275, 2011.

[11] W. A. Hustrulid, "A comparison of laboratory cutting results and actual tunnel boring performace," in N Am Rapid Excav \& Tunnelling Conf. Proc., vol. 2, 1972.

[12] F. F. Roxborough and H. R. Phillips, "Rock excavation by disc cutter," Int. J. of Rock Mech Mining Sci Geomech., vol. 12, pp. 361-366, 1975.

[13] L. Ozdemir, "Development of theoretical equation for predicting tunnel boreability," Ph.D. dissertation, T-1969, Colorado School of Mines, USA, 1977.

[14] H. P. Sanio, "Prediction of the performance of disc cutters in anisotropy rocks," Int. J. of Rock Mech. Mining Sci. Geomech., vol. 22, no. 3, pp. 153-161, 1985.

[15] K. Sato, F. Gong and K. Itakura, "Prediction disc cutter performance using a circular rock cutting ring," in 1st International Mine Mechanization and Automation Symposium, Colorado School of Mines, USA, June, 1991.

[16] J. Rostami, "Design optimization, performance prediction and the economic analysis of TBM application for the construction of proposed Yucca Mountain nuclear waste repository," Ph.D. dissertation, T-3941, Colorado School of Mines, USA, 1991.

[17] J. Rostami and L. Ozdemir, "A new model for performance prediction of hard rock TBM," in Rapid Excavation and Tunnelling Conference, Boston, Soc. for Mining and Metallurgy and Exploration Inc., pp. 793-809, 1993.

[18] L. A. Zadeh, "Fuzzy sets," Inf. Control., vol. 8, pp. 338-53, 1965.

[19] L. A. Zadeh, "Outline of a new approach to the analysis of complex systems and decision processes," IEEE Trans. Syst. Man Cybern., vol. 3, pp. 28-44, 1973.

[20] E. H. Mamdani and S. Assilian, "An experiment in linguistic synthesis of fuzzy controllers," Int. J. Man-Machine Studies, vol. 7, pp. 1-13, 1975. 